\title{
Endoscopic Techniques in Resection of Anterior Skull Base/Paranasal Sinus Malignancies
}

Luke Buchmann, MD; Christopher Larsen, MD; Ania Pollack, MD; Ossama Tawfik, MD, PhD; Kevin Sykes, MPH; Larry A. Hoover, MD, FACS

Objective: The objective of this study was to examine the role of endoscopic approaches to the resection of anterior skull base and paranasal sinus malignancies at one tertiary care medical center. Study Design: The authors conducted a retrospective chart review over a period of 17 years. Methods: Patients undergoing anterior skull base resections for malignancies over a 17-year period were reviewed. Data were collected on each patient with respect to the pathology of the tumor and approach used as well as demographic and follow-up information. Results: A total of 78 patients were treated at a tertiary care medical center for malignancies of the paranasal sinuses and anterior skull base. The most common diagnosis was squamous cell carcinoma occurring in $33 \%$ of the cases. The remaining pathologies included esthesioneuroblastoma (23\%), adenoid cystic carcinoma (15\%), melanoma (3\%), sinonasal undifferentiated carcinoma (3\%), lymphoma (5\%), nasopharyngeal carcinoma (4\%), and other tumor types (14\%). Endoscopic techniques were used extensively in this population of patients. Combined approaches using a sublabial/transmaxillary approach and coronal approaches were used when indicated and complemented the endoscopic approach. A majority of patients were without evidence of disease at the end of this review. Using endoscopic techniques allowed for acceptable cosmetic results and facial incisions were used minimally. Conclusion: With complete endoscopic surgical resection followed by radiation therapy, local recurrence, morbidity, and cosmetic defor-

Presented at the Middle and Western Sections of the Triological Society, February 3, 2006, San Diego, California, U.S.A.

From the Departments of Otolaryngology Head and Neck Surgery (L.B., C.L., K.S., L.A.H.), Neurosurgery (A.P.), and Pathology and Laboratory Medicine (о.т.), University of Kansas Medical Center, Kansas City, Kansas, U.S.A. 7, 2006

Editor's Note: This Manuscript was accepted for publication June

Send correspondence to Dr. Luke Buchmann, University of Kansas Medical Center, Department of Otolaryngology Head and Neck Surgery, 3901 Rainbow Blvd., MS3010, Kansas City, KS 66160, U.S.A. E-mail: lbuchmann@kumc.edu

DOI: 10.1097/01.mlg.0000233528.99562.c2 mity have been minimized. The microscopic view provided by endoscopic techniques, with or without complementary approaches, allows for complete tumor removal. Key Words: Skull base surgery, endoscopic surgery, outcomes, paranasal sinus malignancies, skull base malignancies.

Laryngoscope, 116:1749-1754, 2006

\section{INTRODUCTION}

Anterior skull base malignancies are a challenging problem as a result of the complex anatomy, important structures that may be involved, as well as the skull base fissures and foramina that transmit vessels and nerves and may allow central extension. Until the 1960s, these tumors were often incompletely excised by isolated attempts from either below or above. Extensive lesions in this area often require the combined skills of a team of specialists, including otolaryngologist/head and neck surgeons, skull base surgeons, and neurosurgeons, to successfully resect lesions that extend both intra- and extracranially. Ketcham ${ }^{1}$ in 1963 first described the combined transcranial and transfacial approach to these tumors. Since then, the technologic developments of computed tomography and magnetic resonance image scanning have greatly improved our ability to evaluate the extent of these tumors and plan surgical approaches preoperatively. ${ }^{2}$ When such imaging indicates a tumor is vascular, angiographic techniques and catheters allow embolization to markedly reduce operative bleeding, enhance tumor visualization, and minimize operative blood loss. At the University of Kansas Medical Center, we have organized a skull base surgical team from the previously mentioned surgical specialties. In addition, we frequently seek the help and input of radiation oncologists and medical oncologists who regularly work with us and understand our goals and treatment plan.

\section{PATIENTS AND METHODS}

We conducted a retrospective review of patients who were found to have malignancies of the anterior skull base over a 17-year period (L.A.H.'s tenure at the institution). Slides from all anterior skull base/paranasal sinus cases were then rereviewed by a staff pathologist for confirmation and accuracy of diagnosis. Of 104 pa- 
tients, 78 had sufficient information available to be included in this study for data abstraction, including demographic information, descriptive statistics, and comparative statistics. There was a subset of patients (15) who either had no surgery or received a minimal surgical approach, and these were excluded from the surgical comparison.

A primary objective of our investigation was to evaluate whether the introduction of endoscopic techniques and instrumentation in these cases has resulted in similar outcomes as more classic open surgical approaches, as others have described. ${ }^{3-5}$ Accordingly, the patients were categorized based on outcomes and surgical approach. Outcomes were separated into no evidence of disease, active disease, or death. No evidence of disease was defined as no clinical or radiologic evidence of tumor at the date of the patient's last follow up. Active disease was defined as clinical or radiologic evidence of tumor at the date of last follow up. Categories for surgical approach were established to be able to differentiate between those patients who had a classic open approach for direct visualization and those who had a surgical approach that used cosmetic incisions for access to the anterior skull base with endoscopic techniques and instrumentation for resection.

The goals of the statistical analysis presented in this study were to support the determination of a statistically significant relationship between the outcomes for those receiving the traditional open surgical approach and those receiving an endoscopic approach. Basic calculations of means and standard deviations were used to describe the study population. These means were compared using $t$-tests to determine any statistically significant difference in the surgical groups and the outcome groups. To compare the surgical groups across the outcome results, $\chi^{2}$ tests for heterogeneity were performed. Additionally, Fisher exact tests were used in place of $\chi^{2}$ analysis to compare the distribution of small groups of patients with more advanced disease across the surgical groups.

\section{RESULTS}

Seventy-eight patients were included in this analysis. There were $50(64 \%)$ males and $28(36 \%)$ females with a mean age of 55 years (standard deviation $[\mathrm{SD}]=18$ ). Presentation most commonly included a history of nasal obstruction, sinusitis, or epistaxis that was unilateral. Resected tumors included three main types: squamous cell carcinoma (26), adenoid cystic carcinoma (12), and esthesioneuroblastoma (18). The other histologic tumor types included adenocarcinoma (2), ameloblastoma (1), melanoma (2), nasopharyngeal carcinoma (3), various sarcomas (4), neuroblastoma (1), sinonasal undifferentiated carcinoma (2), small cell neuroendocrine carcinoma (3), lymphomas (3), and metastatic renal cell carcinoma (1) (Table I).

Presentation with advanced disease was common with 56 patients $(72 \%)$ having T3 or T4 tumors and 22 patients (28\%) having T1 or T2 tumors. Outcomes with respect to tumor $\mathrm{T}$ stage were also examined. In patients who died, only $8 \%$ (one of 13) had an initial $\mathrm{T}$ stage of 1 or 2 . T4 tumors had a $69 \%$ mortality rate during the follow-up period, as one might expect.

Follow-up information was available on all patients. Follow up averaged 39 months $(\mathrm{SD}=40)$. Forty-nine $(63 \%)$ of the patients analyzed had no evidence of disease at the time of their last follow up. Sixteen patients (20\%) had active disease at the time of their last follow up, and there were 13 deaths $(17 \%)$. The average follow-up time for the active or deceased group was 35 months $(\mathrm{SD}=38)$ and that of the no evidence of disease group was 41 months $(\mathrm{SD}=41)$. There
TABLE I.

Histologic Tumor Types.

\begin{tabular}{lc}
\hline Histologic Tumor Type & No. \\
\hline Squamous cell carcinoma & $26(33 \%)$ \\
Esthesioneuroblastoma & $18(23 \%)$ \\
Adenoid cystic carcinoma & $12(15 \%)$ \\
Sarcoma & $4(5 \%)$ \\
Nasopharyngeal carcinoma & 3 \\
Small cell neuroendocrine carcinoma & 3 \\
Lymphoma & 3 \\
Melanoma & 2 \\
Sinonasal undifferentiated carcinoma & 2 \\
Adenocarcinoma & 2 \\
Neuroblastoma & 1 \\
Ameloblastoma & 1 \\
Sinonasal undifferentiated carcinoma & 1 \\
\hline \hline
\end{tabular}

was no statistical difference between these two follow-up times $(P=.7)$.

Recurrence occurred in 27 patients (35\%). Treatment methods for recurrent disease included chemotherapy, radiation therapy, and surgery. In general, this occurred in a combined fashion. Seven patients $(27 \%)$ with recurrence received single-modality therapy. Two patients (8\%) received no additional therapy and were treated in a palliative manner. There were $15(58 \%)$ recurrences at the primary site in our patient population. Nine recurrent lesions extended intracranially. Neck recurrences occurred in eight patients $(31 \%)$, and there were three (12\%) instances of distant metastatic disease. Some patients had recurrence at more than one site. Of the 26 patients with recurrent disease, there were six deaths (23\%), $12(46 \%)$ had active disease at the time of last follow up, and, interestingly, eight (31\%) had no evidence of disease at the end of this review period.

Tumor histology also affected outcome. Patients with esthesioneuroblastoma had a good survival rate with only one of 18 (6\%) patients dying during our review period. Patients with squamous cell carcinoma did significantly worse with four of 13 patients (15\%) dying during the same period. Of 12 patients with adenoid cystic carcinoma, two (17\%) also died. T stage at the time of diagnosis was another important factor in overall outcome. Nine of the 13 overall deaths (69\%) had T4 lesions and another three deaths (23\%) had T3 lesions. All together, 92\% of patients who died during this review period had T3 or T4 disease at diagnosis.

Surgical approaches varied in our patient population and evolved over time. To evaluate whether the use of endoscopic techniques affected outcomes, we categorized the surgeries into two groups (Table II). The first were those that did not use endoscopic techniques (external facial incision or Weber-Ferguson and maxillectomy/skull base approaches). The second were those that did use endoscopic techniques (purely transnasal endoscopic approaches; transnasal/sublabial-transmaxillary endoscopic approach; and a combined transnasal/sublabial-transmaxillary endoscopic with frontal craniotomy approach). These two surgical comparison 
TABLE II.

Surgical Categories and Outcomes.

\begin{tabular}{|c|c|c|c|c|c|c|}
\hline Surgical Category & $\begin{array}{l}\text { Surgical } \\
\text { Group }\end{array}$ & Analysis Group & No. of Cases & No Evidence of Disease & Active Disease & Deaths \\
\hline External facial incision & 1 & 1 & 19 & $11(58 \%)$ & $4(21 \%)$ & $4(21 \%)$ \\
\hline Maxillectomy/skull base & 2 & & 8 & $6(75 \%)$ & $0(0 \%)$ & $2(25 \%)$ \\
\hline Endoscopic only & 3 & 2 & 4 & $3(75 \%)$ & $1(25 \%)$ & $0(0 \%)$ \\
\hline $\begin{array}{l}\text { Combined endoscopic and sublabial/ } \\
\text { midface degloving }\end{array}$ & 4 & & 12 & $6(50 \%)$ & $4(33 \%)$ & $2(17 \%)$ \\
\hline
\end{tabular}

groups excluded 15 patients that either had no surgery or some other minimal procedure (leaving 63 surgical patients for comparison). When comparing the distribution of patients with advanced disease (49\% or $78 \%$ ), there was no statistically significant difference between those receiving endoscopic surgery versus nonendoscopic open surgery $(P=$ .36). Data collected on each type of surgical approach is shown in Table III. Thirty-six patients were treated surgically by cosmetic sublabial incisions for access combined with endoscopic surgical resection or endoscopic approach alone. In this surgical group, there were five deaths $(16 \%)$ and eight $(22 \%)$ with active disease. Twenty-three (64\%) had no evidence of disease.

In comparison, 27 patients were treated with classic open facial incisions for direct visualization and resection. In this group, there were six deaths (22\%) and four (15\%) patients with active disease. Seventeen patients $(63 \%)$ had no evidence of disease. $\chi^{2}$ analysis of these outcomes demonstrates that there is no statistical difference in the overall outcome of patients when comparing those who had more classic open approaches with those that had an endoscopic approach $(P=.59)$. Fisher exact test demonstrates that there is no significant difference between the groups with respect to the distribution of $\mathrm{T}$ stage $(P=.36)$ (Table III) Additionally, there is no statistical difference in the mean time of follow up between the two surgical groups $(P=.92)$. The mean follow up for all 63 patients who did receive surgical intervention was 39 months ( $\mathrm{SD}=38$ ). Comparatively, the mean time of follow up for those receiving the nonendoscopic approach was 39 months $(\mathrm{SD}=47)$, whereas those receiving the endoscopic approach had mean time of follow up of 40 months (SD = 31). This data analysis suggests that combining cosmetic incisions for access with endoscopic complete, but sectioned, removal with the microscopic view provided by endoscopes results in just as effective outcomes as open incisions with attempted en bloc resection.

\section{DISCUSSION}

In this retrospective study, we have attempted to review and compare open and endoscopic approaches and treatment outcomes in the resection of anterior skull base/ paranasal sinus malignancies. Our data show that there was an overall no recurrence rate of $67 \%$ in our patient population (33\% recurrence rate). When recurrences happen, they are generally severe and can occur anywhere: locally, intracranially, or at distant sites. In addition, these recurrent lesions are difficult to treat or are fatal (69\% of our recurrences continued to have active disease or died of their disease during this review period). These findings demonstrate the importance of good preoperative evaluation and planning to succeed in a complete resection at the primary surgery if a good long-term outcome is to be achieved.

Tumor histologic type also had a significant impact on survival. The most common tumors were: 1) squamous cell carcinoma (26) of which four (15\%) died; 2) adenoid cystic carcinoma (12), of which two (17\%) died; and 3) esthesioneuroblastoma (18), which had a much better survival with only one of 18 dying during the review period (6\%). Less common tumors included four sarcomas, three of whom are still living. Small cell neuroendocrine carcinomas were rare (3), but two have died of their disease. Finally, of the two patients with sinonasal undifferentiated carcinomas, one died. We have found that combining a cosmetic incision (generally sublabial/transmaxillary for access) with endoscopic resection techniques of tumors is an effective method of approaching these skull base malignancies. Endoscopic visualization of these tumors allows an excellent initial assessment of the tumor and a microscopic evaluation of tumor margins at the end of the procedure for complete resection.

The surgical strategy in this group of patients must be highly individualized because no two tumors are exactly alike. ${ }^{6}$ Tumor grade, aggressiveness, and patient health sta-

TABLE III.

Comparison of Skull Base Surgical Groups.

\begin{tabular}{|c|c|c|c|c|c|}
\hline Surgical Group & Analysis Group & No. $(n=63)$ & No Evidence of Disease & Active & Death \\
\hline 1 and 2 & 1 & 27 (43\%) 85\% T3 or T4 & $17(63 \%)$ & $4(15 \%)$ & $6(22 \%)$ \\
\hline 3,4 , and 5 & 2 & 36 (57\%) $72 \%$ T3 or T4 & 23 (64\%) & $8(22 \%)$ & $5(14 \%)$ \\
\hline
\end{tabular}


tus must also be taken into account when weighing treatment options. In the early portion of this review, combined wide coronal flap anterior craniotomy and lateral rhinotomy (Weber-Ferguson) incisions were commonly used for wide exposure and direct tumor visualization. Over the years, craniotomy size reduced, and endoscopic instrumentation now allows excellent tumor visualization and removal through small cosmetic incisions or by the transnasal route alone. Often, even large anterior skull base tumors can now be removed by subfrontal craniotomy and endoscopic resection of nasal and sinus extensions.

A significant concern in resection of tumors with large extensions into the anterior cranial fossa is watertight closure of large dural and anterior skull base floor defects. In our experience and others, a hardy, carefully dissected pericranial flap provides a rapid, durable, and reliable solution to this problem. ${ }^{7,8}$ Supraorbital, and if possible, superficial temporal, blood supply is cautiously preserved. The pericranial flap is sutured first to the posterior margin of the dural defect, then to the sides, and finally the anterior dural defect margin. This technique provides water- and airtight closure, which is essential for the prevention of cerebrospinal fluid leaks, meningitis, and pneumocephalus. Significant problems have been described when less effective closures are used. ${ }^{9}$ Although pericranial flaps provide excellent closure, protection of the undersurface of these flaps from the hostile environment of the lower nasal cavity is recommended. A splitthickness skin graft of moderate thickness easily adheres to the undersurface of the pericranial flap providing protection from drying and over time contracts to provide a stiff layer that prevents transmitted pulsations.

We have found split-thickness skin grafts also far superior to free bone grafts and metal hardware in medial orbital wall and floor reconstruction. When attempts are made to reconstruct the orbital walls with free bone grafts and metal hardware that are exposed to the hostile environment of a crusting skull base cavity, the invariable result is bone necrosis and extrusion of the metal hardware. This is a problem that can delay important postoperative radiation therapy. On the contrary, split-thickness skin grafts can be anchored to the skull base and orbital bone remnants by sutures sewn through drilled holes. A silk suture "hammock" provides temporary support for the split-thickness skin graft orbital walls until healing of the grafts to orbital soft tissue occurs and contracture pulls orbital structures into their nearly perfect preoperative position.

Lumbar drains have been used fairly consistently in this series, but in recent years for shorter durations and at minimal drainage rates because otherwise significant complications can occur. ${ }^{10}$ When extensive frontal lobe involvement requiring significant frontal lobe resection and/or frontal retraction and trauma is unavoidable, such drainage is very helpful in preventing increased intracranial pressure. When frontal lobe trauma is minimal, however, such drains are rapidly clamped and removed. In all cases, these drains must be monitored very closely. Patient movement and positioning can rapidly increase drainage to alarming levels, and contamination with resultant meningitis is a constant risk.

Finally, a 1-cm-thick layer of "biologic" abdominal fat packing is used to reinforce the pericranial and underlying split-thickness skin graft closure of the anterior cranial floor defect. This aids in the creation of a watertight seal of the defect. A superior portion of this graft will become vascularized and form a permanent portion of the repair. Fibrin glue aids in holding the split-thickness skin graft and underlying fat graft in place and greatly aids in the creation of this watertight seal. ${ }^{11}$ Additional "biologic" abdominal fat packing is placed below this to reinforce the repair, but will not become vascularized and is slowly removed over the first several weeks postoperatively. Below this, nasal trumpets are placed in the inferior meatus and cut to proper length so that they will just break the seal of the soft palate to the posterior pharyngeal wall and stabilized by suturing to the nasal columella. This allows deflation of positive pressure in the nasal cavity and pharynx, especially during coughing and gagging with awakening and extubation, thereby preventing the development of pneumocephalus and air trapping through the fresh anterior skull base repair (a significant problem early on in L.A.H.'s experience).

Skull base procedures can be quite lengthy, and fatigue of team members must be guarded against. Good preoperative planning allows for the surgical excision to be completed in shifts. Generally, the lower portion of the tumor is first excised by the head and neck team members followed by exposure and removal from above. Closure is often the most critical element and should not be left to the most inexperienced members. True skull base tumors are also relatively rare. Although our institution has a regional referral base of over 2 million with little competition for these tumors, we have seen new skull base tumors on average only once every 2 months over this review period, and only $86 \%$ of these were surgical candidates. Such a volume, which is typical for most medium-sized medical centers, has not allowed for one person to focus entirely on the care of skull base tumors. This increases the desirability of using the regularly practiced skills of team members who operate in their respective areas several times a week for other problems. This efficient division of labor allows for rapid and effective tumor removal. The recent technology of image guidance (Fig. 1C), when combined with endoscopic instruments, has greatly improved our ability to perform delicate microscopic dissections at the skull base.

Endoscopic instruments and techniques not only allow excellent visualization of the tumor (Fig. 1C and D), but also greatly aid in their removal. The narrow cavities and recesses of the skull base rarely allow classic en bloc resections as are frequently carried out in the soft tissue of the neck or oral cavity. The mass of large skull base tumors often obscures their site of origin and areas of invasion. Suction debriders designed for polyp removal now allow the rapid excision of the main bulk of large tumors so that small nests and rests of residual tumor can then be identified endoscopically and removed from recesses found throughout the anterior skull base (Fig. 1D). These instruments minimize the need for more radical transbasal approaches that are associated with significantly higher morbidity. ${ }^{12}$ In addition, endoscopic instruments allow wide drainage of sinuses adjacent to anterior skull base resections. If this is not performed, postoperative sinus obstruction frequently occurs and can be the source of serious postoperative infections. ${ }^{13}$ 


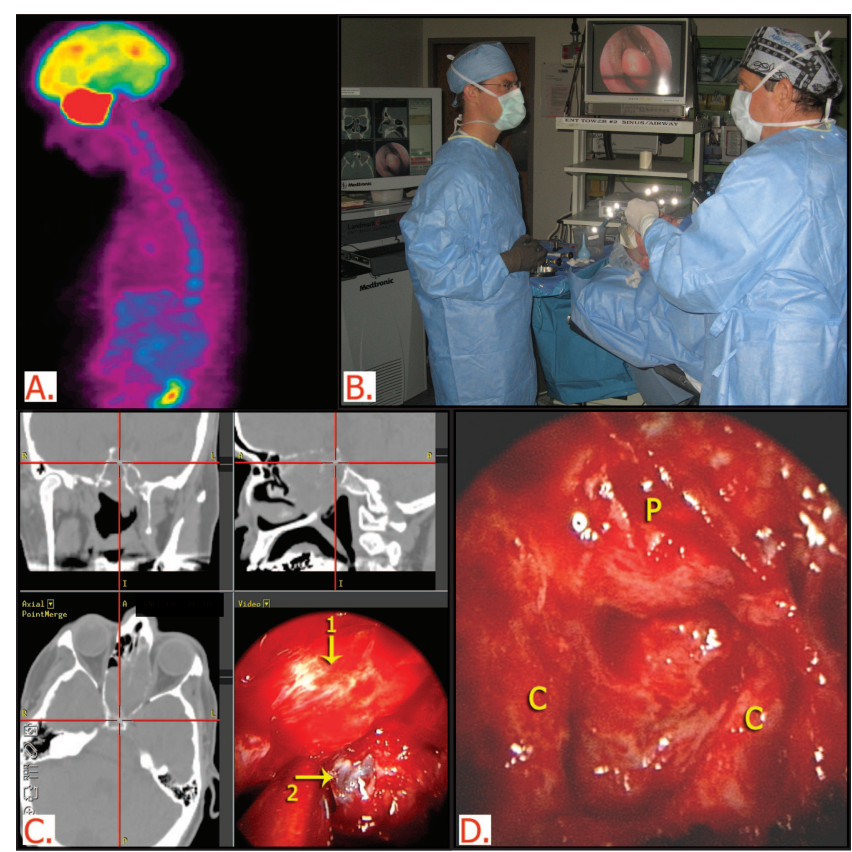

Skull Base Melanoma Case

Legend:

A. Preoperative PET scan demonstrating high signal intensity large skull base melanoma (red). B. Surgical suite with image guidance.

C. Post-operative image guidance with exposed dur a (1) and pituitary (2),

D. Post-operative close-up photo of skull base with skeletonized carotids (C) and pituitary (P)

Fig. 1. Skull base melanoma.

Angiography and embolization of vascular tumors greatly reduce bleeding and allow a dry field in which to use the microscopic view provided by endoscopic telescopes to assure complete removal is accomplished. Although the invasive nature of aggressive squamous cell carcinomas can result in skull base erosion and central invasion, the anatomy of this area favors tumor expansion by the path of least resistance into the nasal and sinus area before actual skull base erosion occurs, and this is especially the case with less aggressive esthesioneuroblastomas and adenoid cystic carcinomas that made up $38 \%$ of these cases. Often, these tumors thin skull base bone by pushing and expanding to the point bone cannot be identified on computed tomography scanning, but after removal, intact skull base or at least intact dura is identified (Fig. 1C). Both of these structures provide effective barriers to central invasion, as does the periorbital membrane surrounding the soft tissues of the eye and the carotid sheath surrounding these vessels (Fig. 1D). Invasion of dura indicates a very aggressive tumor and is associated with high recurrence and failure rates. ${ }^{14}$

Even with the aid of the microscopic view provided by endoscopic telescopes, complete removal of every cell of microscopic disease is often not possible with extensive tumors of the skull base. Complete resection of all of the "visible" tumor as observed by the microscopic view of endoscopic telescopes is a reasonable goal, however. In skull base tumor surgery, it is imperative that reconstructive techniques are used (as described previously) that result in rapid healing so that essential postoperative radiation therapy can be started in a timely fashion. This treatment should begin within 4 weeks of surgery if microscopic residual tumor is to be ster- ilized before resistant larger nodules can be formed. The hostile environment of an anterior skull base surgical cavity probably aids in microscopic residual tumor cell eradication during postoperative radiation therapy. Contrast the nurturing environment of residual tumor cells left behind in a closed neck dissection incision to microscopic disease clinging to life in the hostile environment of a crusting skull base cavity with heavy bacterial colonization.

Patients are examined endoscopically and debrided near the end of radiation therapy and followed especially closely immediately thereafter. Baseline contrasted computed tomography and magnetic resonance imaging scans are obtained 3 months after completion of radiation therapy as a baseline for comparison with future scans. Although our experience with positron emission tomography scanning is too recent and limited for inclusion in this study, it has been very helpful in revealing recurrences or metastases in metabolically active tumors that have a bright signal on preoperative examination (Fig. 1A). On postoperative endoscopic examination, on occasion, small rests of microscopic tumor will form small nodules that can often be removed successfully with forceps and cautery. Larger residual tumor areas of less than $1 \mathrm{~cm}$ can sometimes be eradicated with a boost of focused beam radiation or implantation if they are discovered while still small.

\section{CONCLUSION}

The effective treatment of anterior skull base tumors requires careful pretreatment evaluation with computed tomography, magnetic resonance imaging, and positron emission tomography scanning and preoperative surgical planning by the skull base surgical team. Angiography with embolization can significantly reduce bleeding and improve endoscopic visualization for complete tumor removal with vascular lesions. Combined approaches from above and below now allow complete tumor removal even with extensive lesions with frontal lobe invasion. Watertight closure and prevention of cerebrospinal fluid leaks are attained by carefully suturing a well-vascularized pericranial flap directly into the dural defect. Survival depends not just on the thoroughness of the preoperative evaluation and planning, but the skill of the skull base surgical team. Tumor type greatly affected outcome as did the T stage and, as expected, the extent of skull base tumor invasion. Recurrent tumors are particularly difficult to manage, but nearly one-third of these were salvaged by secondary procedures. The approach to skull base tumors by our surgical team at the University of Kansas Medical Center described in this article has resulted in good survival. Endoscopic surgical techniques have enhanced tumor visualization and precise microscopic resection with outcomes similar to classic open approaches while at the same time decreasing the need for deforming open facial incisions.

\section{BIBLIOGRAPHY}

1. Ketcham AS, Wilkins RH, Vanburen JM, et al. A combined intracranial facial approach to the paranasal sinuses. Am J Surg 1963;106:698-703.

2. Boyle JO, Shah KC, Shah JP. Craniofacial resection for malignant neoplasms of the skull base: an overview. J Surg Oncol 1998;69:275-284.

3. Bockmuhl U, Minovi A, Kratzsch B, et al. [Endonasal micro- 
endoscopic tumor surgery: state of the art.] Laryngorhinootologie 2005;84:884-891.

4. Stammberger H, Anderhuber W, Walch C, et al. Possibilities and limitations of endoscopic management of nasal and paranasal sinus malignancies. Acta Otorhinolaryngol Belg 1999;53:199-205.

5. Thaler ER, Kotapka M, Lanza DC, et al. Endoscopically assisted anterior cranial skull base resection of sinonasal tumors. Am J Rhinol 1999;13:303-310.

6. Lawton MT, Hamilton MG, Beals SP, et al. Radical resection of anterior skull base tumors. Clin Neurosurg 1995;42:43-70.

7. Browne JD, Mims JW. Preservation of olfaction in anterior skull base surgery. Laryngoscope 2000;110:1317-1322.

8. Kelly MB, Waterhouse N, Slade DE, et al. A 5-year review of 71 consecutive anterior skull base tumours. $\mathrm{Br} \mathrm{J}$ Plast Surg 2000;53:184-190.

9. Dias FL, Sa GM, Kligerman J, et al. Prognostic factors and outcome in craniofacial surgery for malignant cutaneous tumors involving the anterior skull base. Arch Otolaryngol Head Neck Surg 1997;123:738-742.

10. Kryzanski JT, Annino DJ Jr, Heilman CB. Complication avoidance in the treatment of malignant tumors of the skull base. Neurosurg Focus 2002;12:e11.

11. Chandler JP, Silva FE. Extended transbasal approach to skull base tumors. Technical nuances and review of the literature. Oncology 2005;19:913-919; discussion 920, 923-925, 929.

12. Feiz-Erfan I, Han PP, Spetzler RF, et al. The radical transbasal approach for resection of anterior and midline skull base lesions. J Neurosurg 2005;103:485-490.

13. Morioka M, Hamada J, Yano S, et al. Frontal skull base surgery combined with endonasal endoscopic sinus surgery. Surg Neurol 2005;64:44-49; discussion 49.

14. Ichimura K, Sasaki T, Nakatsuka T, et al. Analysis of tumor recurrence following anterior skull base surgery. Eur Arch Otorhinolaryngol 1998;255:155-162. 